\title{
Effect of Indirect Neural Decompression by Minimally Invasive Oblique Lumbar Interbody Fusion in Adult Degenerative Lumbar Spine Disease and Its Limitations
}

\author{
Nisarg P. Parikh ${ }^{1}$, Manish Mistry², Amit Chandrakant Jhala ${ }^{2}$ \\ ${ }^{1}$ Swasti Spine and Mind Care, Ahmedabad, '2 Chirayu Orthopedic Fracture and Spine Hospital, Ahmedabad, India
}

Corresponding Author:

Amit Chandrakant Jhala, MS, DNB

Consultant Spine Surgeon, Chirayu

Orthopedic Fracture and Spine Hospital,

203-204, Chirayu Complex, Narayan

Nagar Road, Paldi Ahmedabad, Gujarat

380007, India

Tel: +91-9825040172

E-mail: acjhala@gmail.com

Received: February 6, 2019

Revised: April 11, 2019

Accepted: May 28, 2019
Objective: To study efficacy of minimally invasive oblique lumbar interbody fusion (MIS OLIF) to achieve indirect decompression in degenerative lumbar spine disorders. To find out amount of indirect decompression achieved by assessing clinical and radiological outcomes. To find out limitations of indirect decompression. Methods: OLIF was carried out in 60 segments/45 patients having degenerative lumbar spine disorders from May 2016 to April 2018. Patients with infection, trauma, lumbar disc prolapse and listhesis > grade 3 were excluded. 53 segments had posterior and 7 segments had anterior fixation. Auto graft was used in 21 and artificial bone graft in 24 patients. Indirect decompression by MIS OLIF was achieved in all patients. Neuromonitioring was not used. Clinical assessment was done using Modified Macnab's criteria. Radiological assessment was done on X-rays and MRI. Percentage improvement in foraminal height, disc height, segmental lordosis, spinal canal area and reduction in listhesis were measured. Statistical assessment was done using Paired't' test. Results: 60 segments of 45 consecutive patients were operated with 15 of them male and 30 female. Average age was 63 years. Minimum follow-up was for 1 month and maximum follow-up was for 18 months with average of 11 months. Single segment fusion was done in 31, 2 segment fusion in 13 and 3 segment fusion in 1 patient. Clinically 33 (73.33\%) had excellent, 11 (24.44\%) had good \& 1 (2.22\%) had fair outcomes. None required direct decompression. Radiologically; foraminal height improved by $26.27 \%$, disc height $92.1 \%$, segmental lordosis $3.4^{\circ}$ and listhesis reduction was $6.8^{\circ}, 41 \mathrm{seg}-$ ments studied on MRI had improvement in spinal canal area of $42.7 \%$. Conclusion: Indirect decompression by MIS OLIF is effective in decompressing the spinal canal with good radiological and clinical out comes. Direct decompression is avoidable with help of interbody distraction using OLIF particularly in patients with Schizas grade $A, B$, and C of lumbar spinal stenosis.

Key Words: Indirect decompression, OLIF, Degenerative lumbar spine

\section{INTRODUCTION}

Lumbar spinal stenosis as a result of the hypertrophy of the articular process was first described by Verbiest in $1954^{1)}$. It has two components viz. Static and dynamic. It is known that the changes in acquired lumbar spinal stenosis are because of the soft tissue as well as bony components of the spinal canal. The cascade which affects the facet joints as well as disc and other soft tissues are well depicted by Kilkardy Willis ${ }^{2}$. Due to ageing process and repetitive loading and unloading of the spine there is disc degeneration and facet joint osteoarthritis which is a pathoanatomical feature associated with sagittal orientation of facets and degenerative spondylolysthesis ${ }^{3,4)}$. These changes will make spine more unstable and increase mechanical stresses on the ligamentum flavum which causes its hypertrophy ${ }^{5,6)}$. All these changes ultimately lead to narrowing of the canal and encroachment on its contents. The patients in the unstable phase of this cascade will clinically present with neurogenic claudication without rest pain. Symptomatic patients with unstable spine will require stabilization along with decompression. As a conventional mode of treatment they are treated with direct decompression along with stabilization. However with the recent views, these subset of patients can be treated 
with indirect decompression through restoration of the disc height and foraminal height by ligamentotaxis effect of distraction of the disc space. This can help us avoid all the complications which are related with conventional posterior direct decompression and interbody fusion including more muscle damage, bony resection of lamina and facets, bleeding, nerve root injuries, dural tear, epidural bleeding, hematoma, deep wound infection, perineural fibrosis etc. Various studies have described efficacy of anterior approach like ALIF and lateral approaches like XLIF/ LLIF and OLIF to decompress the neural elements indirectly ${ }^{7-17)}$. Transpsoas approach (LLIF/XLIF) is associated with higher rates of approach related neurological complications due to injury to lumbar complex. There is reported neurological complication rate of $3-33 \%$ in literature ${ }^{18)}$. ALIF is possible only at L5-S1 level where as OLIF can be done at all lumbar segments. Also, ALIF is done through open retroperitoneal approach which has higher approach related morbidities as compared to OLIF which can be done through minimal access approach.

Minimally Invasive OLIF has several advantages like less blood loss, shorter hospital stay, less back pain and faster recovery; all in comparison to posterior direct approaches. It is a retroperitoneal approach which uses an oblique corridor between great vessels anteriorly and psoas posteriorly; first described by Michael Mayer in $1997^{19,20)}$. It retains all the advantages of anterior approaches viz. restoration of disc height, opening up of the foramen and restoration of segmental lordosis with the help of ligamentotaxis, keeping ALL and PLL intact which are major stabilizing structures of spine ${ }^{21,22)}$. As it is an anterior to psoas approach it avoids complications occurring because of lumbar plexus injuries like paresthesia of anterior thigh, psoas weakness etc ${ }^{23,24)}$. Hence, OLIF can be done without neuromonitoring as well.

This study focuses on efficacy of minimal invasive (OLIF) to stabilize the spine and to achieve indirect neural decompression in degenerative lumbar spine disorders. However it has not been clearly understood in which cases we can avoid direct decompression. In this study we have quantified the amount of indirect decompression OLIF can achieve and in which cases it is feasible to use them by assessing clinical and preoperative and postoperative radiological parameters like changes in disc height, foramen height, spinal canal area as well as restoration of segmental lordosis.

\section{Aim of the Study}

* To assess efficacy of OLIF to achieve indirect decompression in degenerative lumbar spine disorders.

* Quantify the amount of indirect decompression achieved by assessing clinical outcomes and radiological parameters.

* Limitations of indirect decompression.

* To assess the patients who required direct decompression after performing OLIF.

\section{MATERIALS AND METHOD}

Retrospective analysis of 45 consecutive patients was done from May 2016 to April 2018 in whom minimally invasive oblique lumbar interbody fusion was carried out. These patients had in total 60 segments operated.

Inclusion criteria were failed conservative line of management for low back pain with or without radicular pain and neurogenic claudication in lower limbs which got relieved with rest. Exclusion criteria were rest pain, infection, trauma, spondylolisthesis more than grade 3 and acute lumbar disc prolapse. All included patients were counselled for the requirement of direct decompression in case of persistent symptoms after OLIF.

Clinical and neurological assessment of all the patients was done. Radiological analysis was done using standardized plain $\mathrm{AP}$ and Lateral dynamic X-rays and MRI of lumbosacral spine. Appropriate size of cage was inserted using retroperitoneal oblique corridor under IITV guidance. Bone graft was also used along with cage. Autograft was used in 21 patients and artificial bone graft with bone marrow aspirate was used in 24 patients. After cage insertion, fixation of the lumbar segment was done in the same stage. Posterior percutaneous fixation was done in 53 whereas anterior fixation was done in 7 segments. No direct decompression was done. No Neuromonitoring was carried out. Perioperative complications were noted and follow-up was done at 1,3 , and 6 months and at 1 year. Postoperative clinical assessment was done using Modified Macnab's criteria.

Postoperative $\mathrm{X}$-rays of lumbosacral spine AP and lateral view were taken in all patients. $\mathrm{X}$-rays were taken at 1 month, 3 months, 6 months and 1 year follow-up. Immediate postoperative MRI was studied in 41 segments. All these segments were divided into two groups depending on Schizas grading ${ }^{25}$ of lumbar spinal stenosis. Group 1 had class A+B patients whereas group 2 had class $C+D$ patients. Group 1 had 25 segments of 21 patients and group 2 had 16 segments of 16 patients. Figure 1 enumerates the Schizas classification of lumbar spinal stenosis. In grade A stenosis: there is clearly CSF visible inside the dural sac, but its distribution is inhomogeneous. Grade B stenosis:
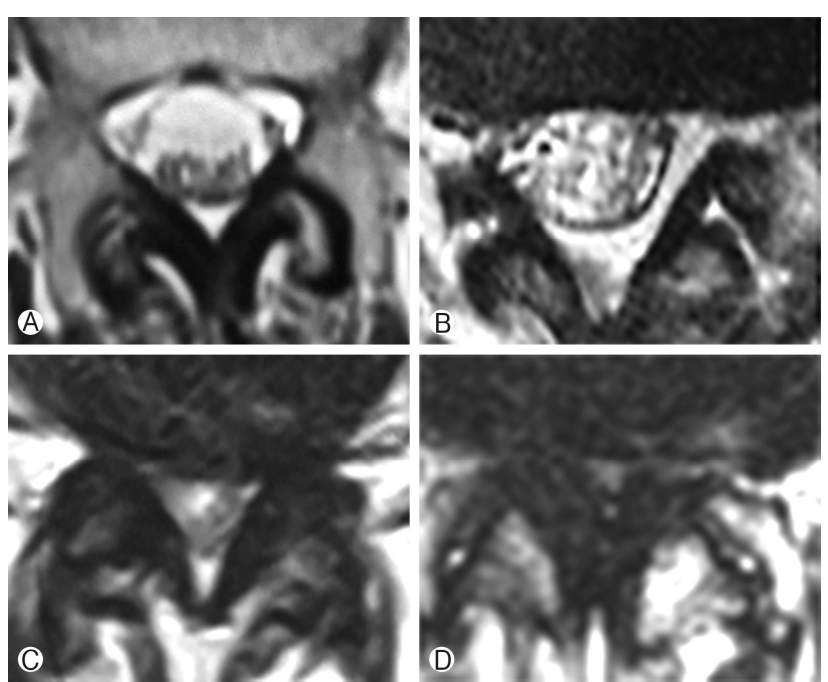

Fig. 1. Schizas Grading. (A) Schizas grade A. (B) Schizas grade B. (C) Schizas grade C. (D) Schizas grade D. 

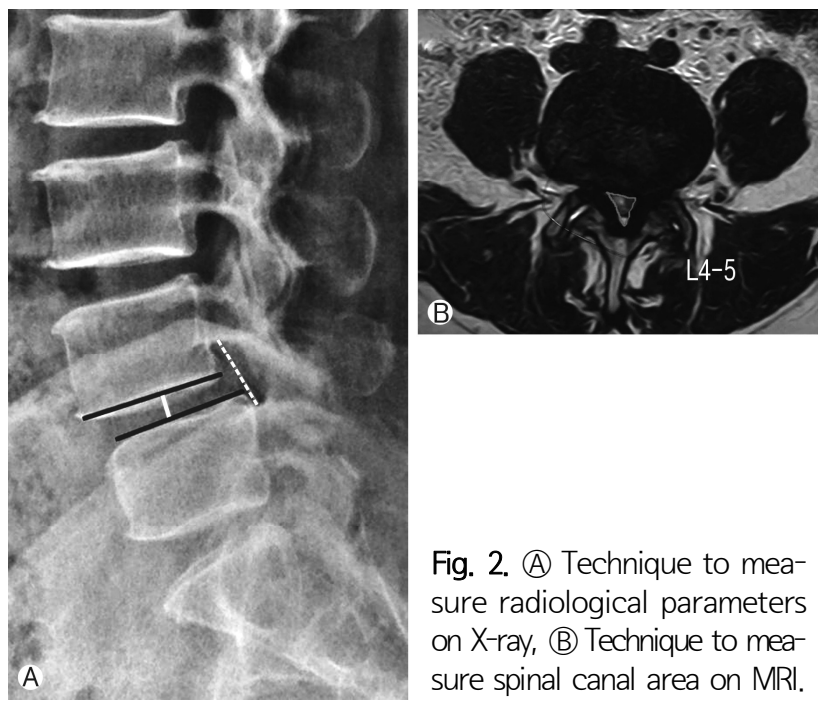

Fig. 2. (A) Technique to measure radiological parameters on X-ray, (B) Technique to measure spinal canal area on MRI.

the rootlets occupy the whole of the dural sac, but they can still be individualized. Some CSF is still present giving a grainy appearance to the sac. Grade $\mathrm{C}$ stenosis: no rootlets can be recognized, the dural sac demonstrates homogeneous gray signal with no CSF signal is visible. There is epidural fat present posteriorly. Grade D stenosis: in addition to no rootlets being recognizable there is no epidural fat posteriorly.

Assessment of radiological parameters: Percentage improvement in disc height, foraminal height, segmental lordosis on $\mathrm{X}$-rays, reduction of slippage of vertebrae and increase in overall area of spinal canal area were noted to assess indirect decompression. All results were statistically analyzed using 'paired T' test. Surgimap was used for calculation of parameters. To calculate the disc height a perpendicular line was drawn from the midpoint of cranial vertebra of the respective disc space to the caudal endplate and this length was taken as disc height. Segmental lordosis was measured as the sagittal Cobb's angle of the cranial and caudal endplate of the involved disc. Foraminal height was also measured between cranial most and caudal most point of the foramen on true lateral $X$-ray lumbosacral spine. Spinal canal area was measured on MRI on single axial slice through the center of the disc. Outlines of the spinal canal area were mapped manually on the software and the area enclosed was measured (Fig. 2). Pre-op and post-op comparisons of all these parameters were done and percentage improvement was calculated. Measurements were taken by two surgeons and average of it was taken as the final measurement.

\section{OLIF Procedure}

* The patient was positioned in a true right lateral decubitus position. A 4- to 5-cm skin incision 3-4 $\mathrm{cm}$ anterior to the desired disc space after marking under IITV, parallel to the fibers of the external oblique muscle, was put. The approach was usually performed from the left side but could also be performed from the right side, especially in patients who had previously received the procedure from the left side.

* The external oblique, internal oblique and transverse abdominal muscles were then dissected along the direction of their fibers.

* The retroperitoneal space was accessed by blunt dissection, and the peritoneal content was mobilized anteriorly. The psoas muscle and genitofemoral nerve were identified, and the intervertebral disc was exposed through an open corridor between the psoas muscle posteriorly and great vessels anteriorly.

* First dilator was placed over the disc space at junction of anterior $1 / 3^{\text {rd }}$ and posterior $2 / 3^{\text {rd }}$, which was identified fluoroscopically. A guide wire was then introduced through the first dilator into the disc space. The first dilator was then hammered inside the disc space and the guide wire was removed. Sequential dilators were placed over the first dilator. Expandable tubular retractor of appropriate size was placed over the final dilator and docked on to the disc space.

* A distractor pin was used which also served to retain the tubular retractor in place over the disc space.

* Annulotomy was done over the disc and, the disc material including the cartilaginous endplate was excised sequentially by rongeurs, shavers and curettes. The annulus fibrosis at the opposite side was released completely with caution using Cobb elevator under fluoroscopic viewing. Orthogonal maneuver was used for all the instruments for disc preparation inside the disc space. The psoas muscle was retracted temporally during the maneuver.

* Cage size was determined by appropriate sizers, which were inserted into disc space and confirmed on IITV images.

* An appropriate-sized cage was filled with autologous bone graft from the iliac crest or synthetic bone substitute and was inserted in a press-fit fashion into the disc space. Because these maneuvers are performed anterior to psoas muscle and the retraction was gentle and temporary, neuromonitoring was not needed. Upon insertion of cage either anterior fixation was done or the patient was turned to the prone position, and supplemental percutaneous posterior pedicle screws were placed. Compression was done.

\section{RESULTS}

\section{Demographic Characteristics}

60 segments of 45 consecutive patients ( 15 males and 30 females) were operated. Average age was 63 years. Minimum follow-up was for 1 month and maximum follow-up was for 18 months with average of 11 months. Thirty one patients had degenerative listhesis, 2 had disc degeneration, 3 had lytic listhesis, 4 had lumbar canal stenosis without instability noted on dynamic $X$-rays and 5 had adjacent segment disease. Single segment fusion was done in 31 patients, double level fusion 
done in 13 patients whereas 3 segment fusion done in 1 patient. Average estimated duration for single segment cage placement was 40 minutes (Table 1).

Complications: There were some approach related complications. Major per-operative complication was rupture of the iliolumbar vein in $1(2.22 \%)$ patient. However it was effectively controlled with help of hemocoagulants and without any post-op morbidity. Other per-op complications included peritoneumbreach in $2(4.44 \%)$ patients which was sutured and fracture of the superior endplate or inferior endplates in $2(4.44 \%)$ patients during cage preparation who were severely osteoporotic. Per-op complication rate was $11 \%$.

Post operative complications were: One patient $(2.44 \%)$ had

Table 1. Demographic characteristics

\begin{tabular}{lc}
\hline \hline Total patients/segments & $60 / 45$ \\
\hline Sex; Male/Female & $15 / 30$ \\
Age & $40-82$ years; 63 years \\
Average follow-up & 11 months \\
Diagnosis & No. of patients \\
Degenerative listhesis & 31 \\
Disc degeneration & 2 \\
Lytic listhesis & 3 \\
Lumbar canal stenosis & 4 \\
Adjacent segment disease & 5 \\
Levels of fusion & No. of patients \\
Single segment & 31 \\
Two segment & 13 \\
Three segments & 1 \\
Fused levels & \\
L1-2 & 3 \\
L2-3 & 4 \\
L3-4 & 16 \\
L4-5 & 36 \\
L5-S1 & 1 \\
\hline
\end{tabular}

Table 2. Clinical results

\begin{tabular}{lc}
\hline \hline Excellent & $33(73.33 \%)$ \\
Good & $11(24.44 \%)$ \\
Fair & $1(2.22 \%)$ \\
\hline
\end{tabular}

superficial and 1 patient (2.44\%) had deep infection which got resolved with oral antibiotics. Two patients (4.44\%) had numbness in area distribution of genitofemoral nerve. Other notable complication was contralateral radiculopathy which was present in $7(15.55 \%)$ patients. Overall rate of postoperative complication was $33 \%$. Noteworthy is the fact that most of these complications were reversible. Autograft was taken in 21 patients. Amongst them $11(24.44 \%)$ had hip flexion weakness due to pain. However all of them recovered within 2-8 weeks with average recovery of 6 weeks. Three $(6.67 \%)$ patients had fracture of the anterior iliac spine which got healed with rest. None had ureteric or permanent neurologic deficit. None of the patients required direct decompression.

Clinical Results: Clinical assessment was done according to Modified Macnab's criteria. Thirty three (73.33\%) patients had excellent result, $11(24.44 \%)$ had good result and 1 (2.22\%) had fair result (Table 2, Chart 1). Overall 98\% patients had excellent to good clinical outcome.

Average hospital stay of the patients was 2 days. On an average, the patients resumed their normal activities within 3 weeks of surgery.

\section{Radiological Results:}

The following parameters were studied either on X-rays or MRI:

* foraminal height

* disc height

* reduction in vertebra slippage (listhesis)

* Improvement in segmental lordosis

* Spinal canal area

Total 41 segments were studied on MRI. The rest of the segments could not be studied well because of the artifact effect. Comparison of pre-operative and post-operative data was done and mean of percentage improvement was calculated (Table 3).

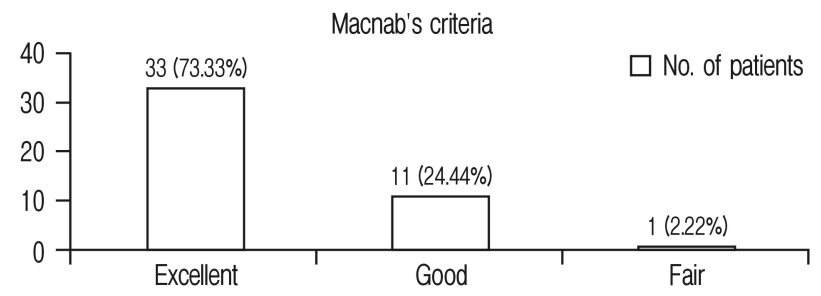

Chart 1. Macnab's criteria.

Table 3. Radiological outcomes

\begin{tabular}{lcccc}
\hline \hline Radiological parameters & Mean of improvement & 'P’ Value & T value & Degree of freedom \\
\hline Foraminal height & $26.27 \%$ & $<0.0001$ & 7.43 & 36 \\
Disc height & $92.1 \%$ & $<0.001$ & 10.38 & 36 \\
Segmental lordosis & $3.4^{\circ}$ & $<0.001$ & 3.89 & 26 \\
Listhesis reduction & $6.8^{\circ}$ & $<0.0001$ & 5.07 & 36 \\
Spinal canal area & $42.7 \%$ & $<0.0001$ & 6.16 & 29 \\
\hline
\end{tabular}


All the segments were divided into two groups, depending upon Schizas MRI grading of lumbar canal stenosis, and improvement in spinal canal area was compared between two groups.

Improvement in foraminal height was $26.27 \%$ and has been shown in Figure 3 and Chart 2.

Improvement in disc height was $92.1 \%$ and has been shown in Figure 4 and Chart 3.

Improvement in listhesis reduction was $6.8^{\circ}$ and has been shown in Figure 5 and Chart 4.

Improvement in segmental lordosis was $3.4^{\circ}$ and has been shown in Figure 6 and Chart 5. It was noted that if the cage position
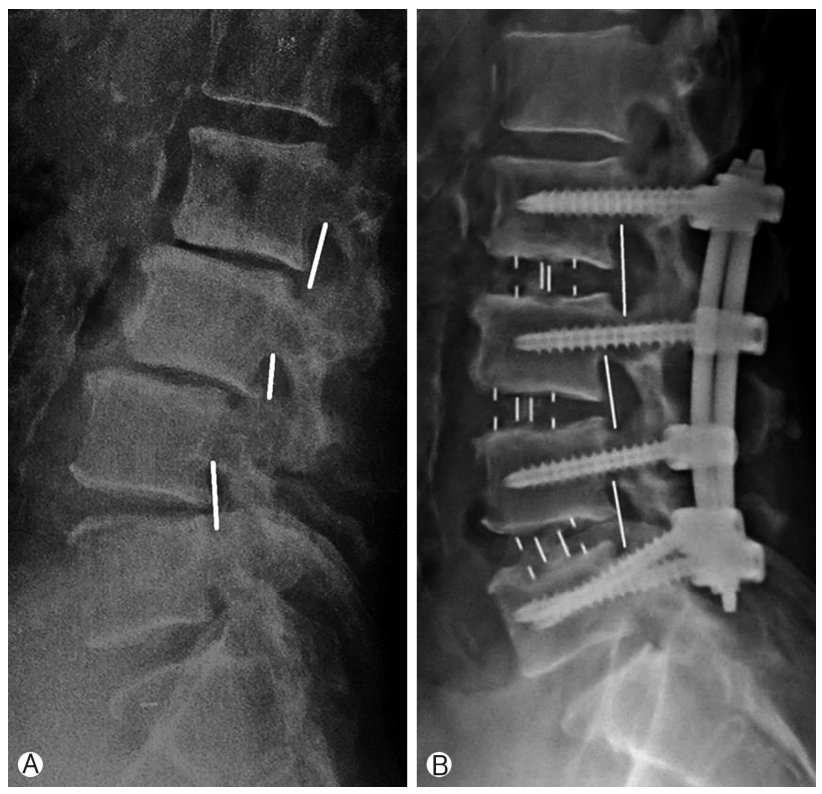

Fig. 3. (A) (B) Increase in foraminal height at $L 2-3, L 3-4, L 4-5$ levels after OLIF.

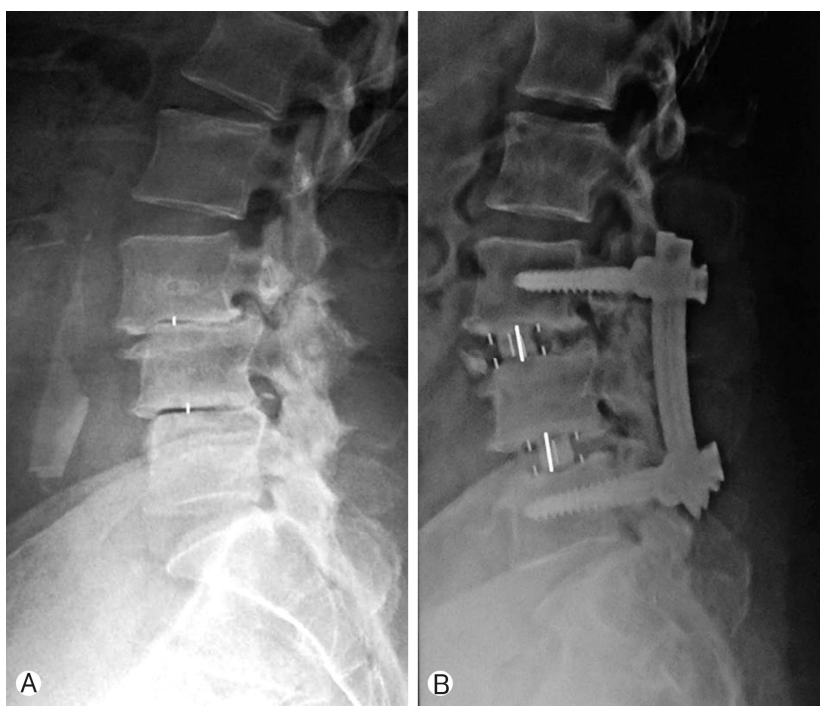

Fig. 4. (A, B Restoration of Disc height at $L 3-4$ and $L 4-5$ is remarkable after OLIF.

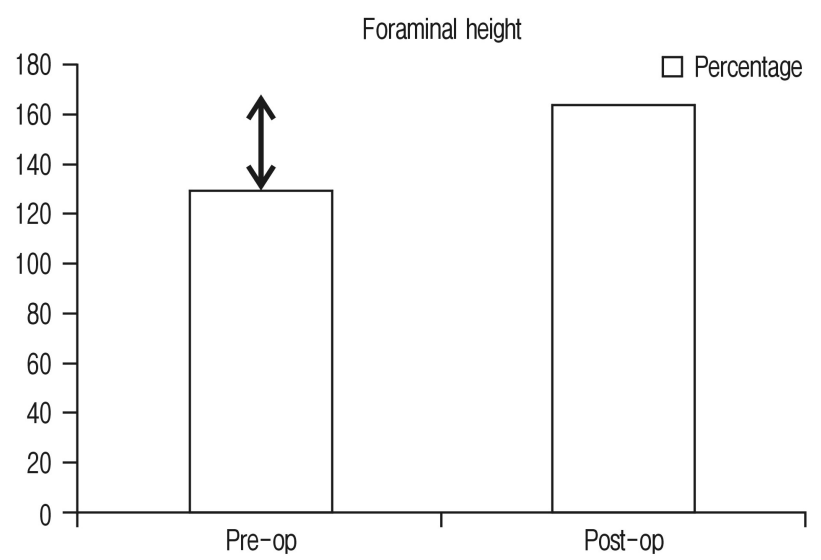

Chart 2. Foraminal height.

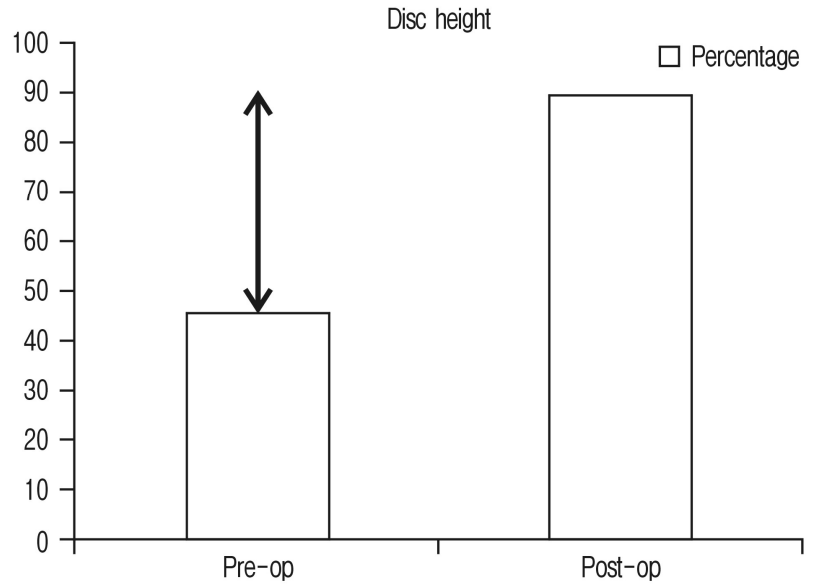

Chart 3. Disc height.
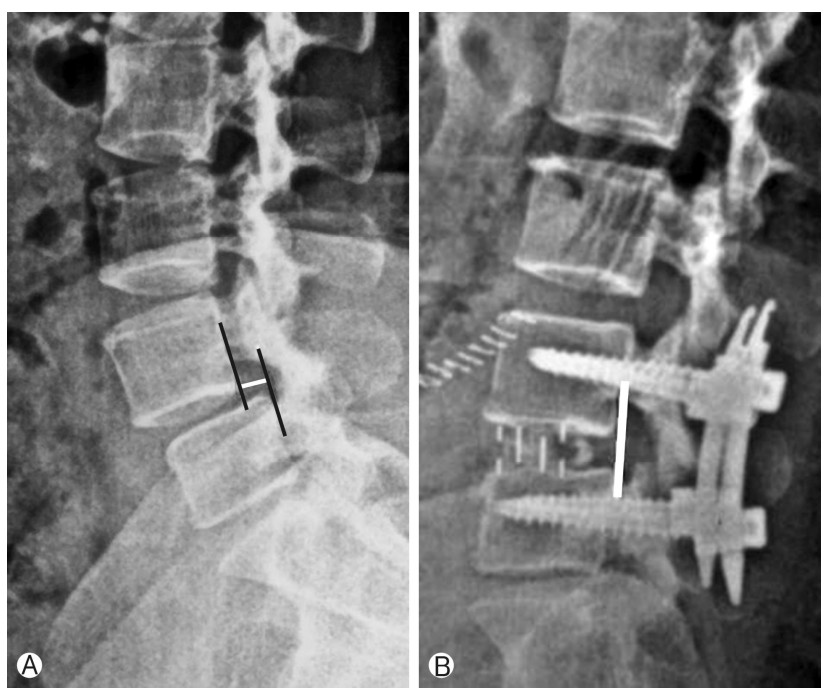

Fig. 5. (A) (B) Reduction in slippage(lystheis) at L4-5 after OLIF. 


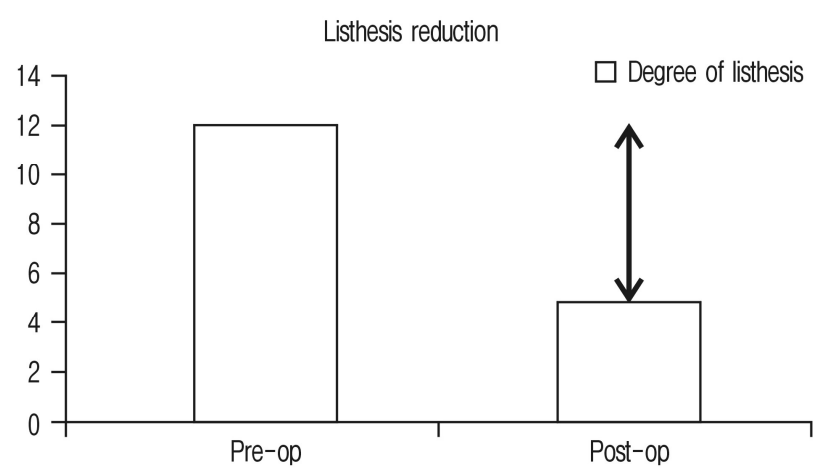

Chart 4. Listhesis reduction.
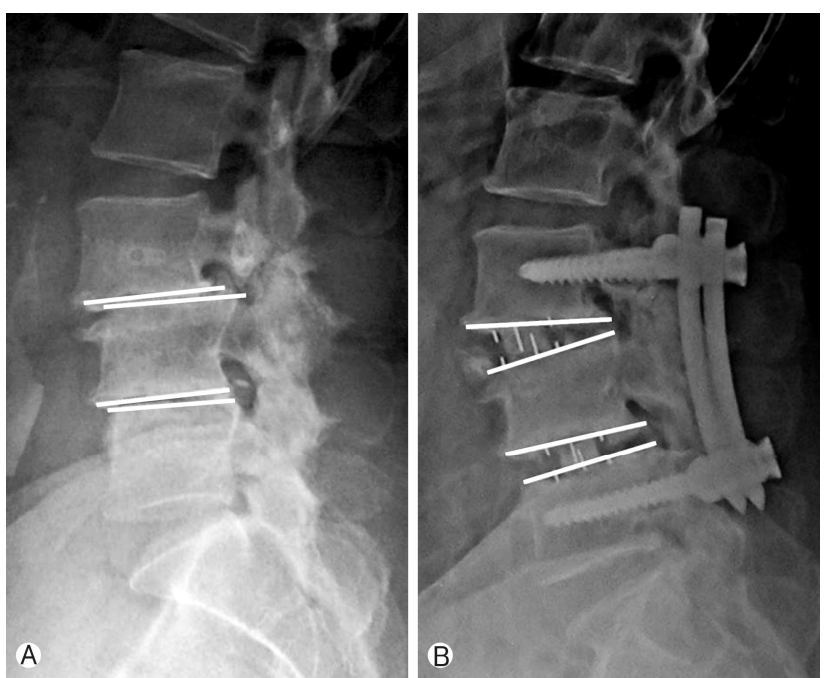

Fig. 6. (A), B Restoration of the segmental lordosis at operated levels. Cage position can influence lordosis achieved.
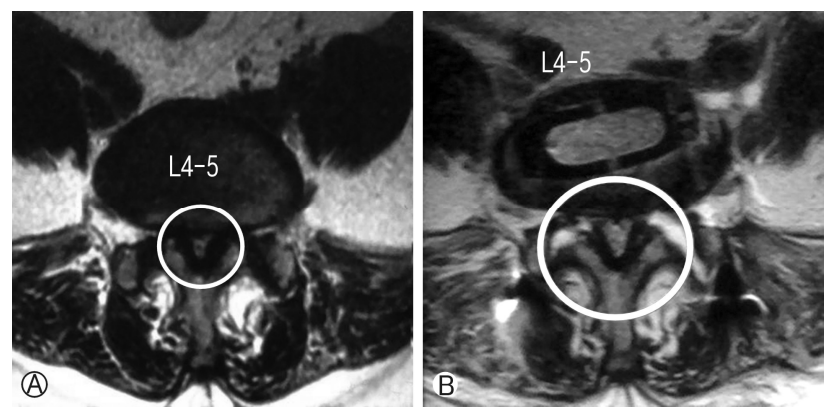

Fig. 7. (A), B) Increase in overall spinal canal area postoperatively after OLIF. were more anterior, it would achieve more segmental lordosis whereas middle or posterior position would have lesser improvement in segmental lordosis.

Improvement in spinal canal area was $42.7 \%$ overall and has been shown in Figure 7 and Chart 6.

Group 1 had 25 segments of 21 patients. Group 2 had 16 segments of 16 patients. Radiologically group 1 had spinal canal area improvement of $26.57 \%$ whereas group 2 had spinal canal area improvement of $72.78 \%$ (Table 4). Clinically all the patients of group (100\%) showed excellent to good outcome, as per Modified Macnab's criteria. Amongst group 2 patients, only 1 patient $(6.25 \%)$ showed fair outcome whereas the rest of the patients (93.75\%) showed excellent to good outcome as per Modified Macnab's criteria.

\section{Case Example}

Fifty years old male patient presented with complaints of lower limb (right>left) neurogenic claudication for more than a year. Walking distance was $<500$ meters. Standing time was 5 minutes. There was no resting pain. Examination showed normal neuro-

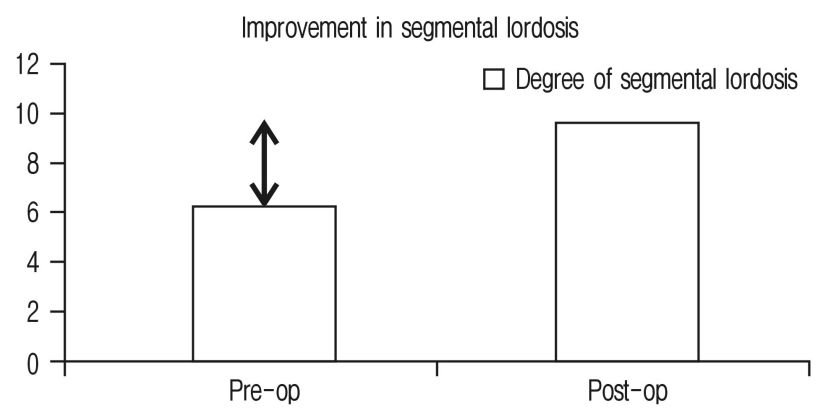

Chart 5. Improvement in segmental lordosis.

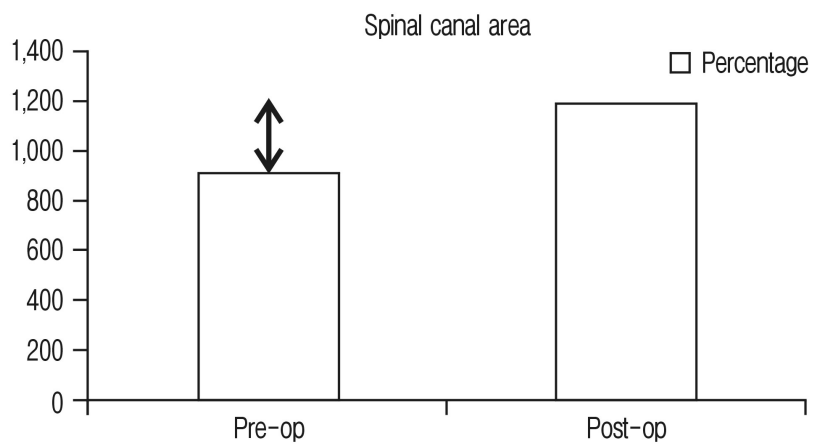

Chart 6. Spinal canal area.

Table 4. Segments on MRI as per Schizas grade

\begin{tabular}{lcc}
\hline \hline & No. of segments & Spinal canal Improvement \\
\hline Group 1 (Schizas class A+B) & 25 & $26.57 \%$ \\
Group 2 (Schizas class C+D) & 16 & $72.78 \%$ \\
\hline
\end{tabular}


logy. Radiology showed degenerative spondylolisthesis at L3-4 and L4-5 levels with lumbar canal stenosis on MRI (Schizas grade $B$ at L3-4 and grade C at L4-5). Minimal access OLIF was performed at both the levels and posterior percutaneous fixation was done. The patient clinically improved and resumed his normal activities within 3 weeks. Clinically the patient showed Macnab grade $\mathrm{A}$ improvement. Also there was radiological improvement in foraminal height, disc height, spinal canal area and segmental lordosis postoperatively (Fig. 8).

\section{DISCUSSION}

The current study shows the efficacy of OLIF to achieve indirect neural decompression in degenerative lumbar spine. The study included patients of degenerative lumbar spine disorders who had symptoms of neurologic claudication. None of them had rest pain. None of the patients were clinically positive for signs of nerve root tension. None required direct decompression. Excellent to good clinical results were achieved in $98 \%$ of the patients, while 1 had fair result. This patient had developed contralateral radiculopathy postoperatively due to cage malposition. Although he developed opposite sided leg pain, he was free from preoperative symptoms of neurogenic claudication.
Radiologically there were improvements in disc height (92.1\%), foraminal height (26.27\%), spinal canal area (42.7\%), and segmental lordosis $\left(3.4^{\circ}\right)$ which was statistically significant. These results show that in these patients direct decompression could be avoided and so all the potential complications of directdecompression like amount of back pain pertaining to muscle dissection or bony resection of lamina and facets, bleeding, nerve root injuries, dural tear, epidural bleeding, hematoma, deep wound infection etc., were avoided.

The concept of indirect neural decompression was introduced via ALIF procedure in patients of lumbar disc herniation ${ }^{77}$. There have been numerous other articles published as well which state that indirect neural decompression which can be achieved through ALIF, OLIF or LLIF/XLIF is beneficial in degenerative unstable lumbar spine disorders ${ }^{8-17)}$. However none of these studies has defined the amount of radiological indirect decompression required for clinical improvement of patients as well as its limitations. The placement of the larger interbody cages through anterior approach is at the center of the philosophy of indirect decompression. It leads to better restoration of the disc height, unbuckling and stretching of the ligamentum flavum, foraminal opening and reduction of the posterior disc bulge which in turn decompresses the neural structures ${ }^{26)}$. However
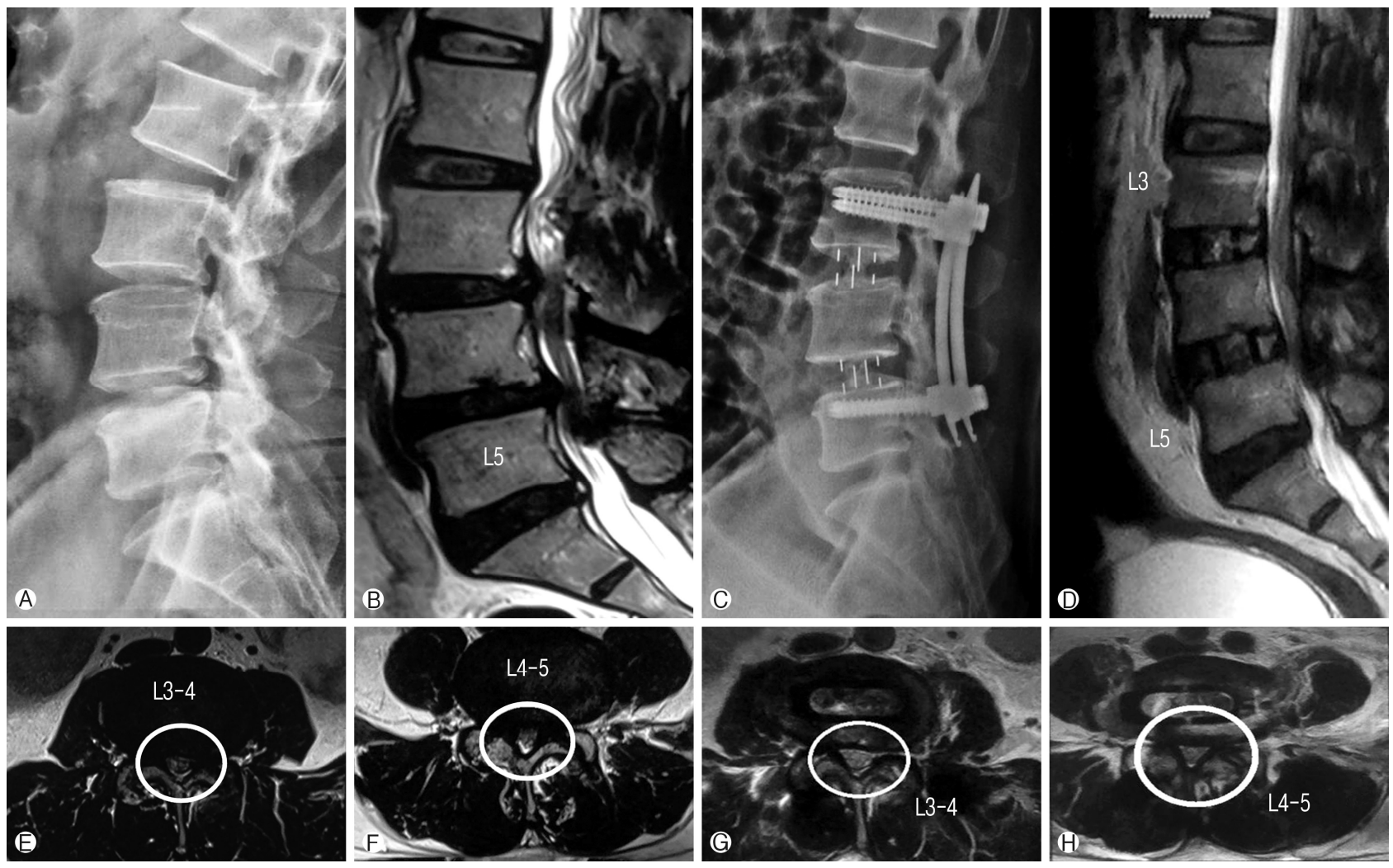

Fig. 8. (A) Preoperative LS Spine Lateral X-ray. (B) Preoperative LS Spine T2W sagittal MRI showing stenosis at L3-4 and L4-5. (E) and (F) Axial T2W MRI shows stenosis at L3-4 and L4-5 levels with subluxated facets at L3-4. (C) and (D) Postoperative Lateral X-ray and T2W sagittal MRI shows restoration of disc height, foraminal height and segmental lordosis at operated levels as compared to preoperative images. (G) and $\mathbb{A}$ Postoperative T2W axial MRI shows increase in the overall spinal canal area compared to preoperative images as well as cage positioning. 
Nisarg P. Parikh, et al.

Table 5. Various studies showing efficacy of indirect decompression

\begin{tabular}{|c|c|c|c|c|c|c|}
\hline & $\begin{array}{c}\text { Sample size } \\
\text { (segments) }\end{array}$ & Procedure & $\begin{array}{l}\text { Disc height } \\
\text { improvement }\end{array}$ & $\begin{array}{c}\text { Foraminal height } \\
\text { improvement }\end{array}$ & $\begin{array}{c}\text { Spinal canal area } \\
\text { improvement }\end{array}$ & $\begin{array}{c}\text { Direct } \\
\text { decompression }\end{array}$ \\
\hline Oliveira et al. ${ }^{8)}$ & 43 & XLIF & $41.9 \%$ & $13.5 \%$ & $33 \%$ & 2 patients \\
\hline Jun Sato et al. ${ }^{28)}$ & 20 & OLIF & $61.0 \%$ & $18 \%$ & $19 \%$ & None \\
\hline Fujibayashi et al. ${ }^{29)}$ & 52 & OLIF & $82.3 \%$ & - & $30.2 \%$ & None \\
\hline Marjan Alimi et al. ${ }^{30)}$ & 145 & LLIF & $82.9 \%$ & $20.12 \%$ & - & None \\
\hline Our study & 60 & OLIF & $92.1 \%$ & $26.27 \%$ & $42.7 \%$ & None \\
\hline
\end{tabular}

this doesn't cause direct thinning of the ligamentum flavum. It is known that unstable spine leads to cascade of degenerative changes affecting the spinal structures and also thickening of the ligamentum flavum. Sehji Ohtori et al. ${ }^{26)}$ in their study of 10 years follow-up of ALIF patients showed that stabilization of unstable spine leads to remodeling of spine and ligamentum flavum as well. All the segments, included in their study, had reduced thickness of ligamentum flavum as compared to immediate postop period, and spinal canal area was also larger.

As discussed earlier, all the anterior approaches including XLIF, ALIF, and OLIF allow placement of larger interbody cages as compared to posterior approaches. This leads to greater restoration of disc height and thus indirectly decompresses the spinal canal. Groth et al. ${ }^{27)}$ described similar changes after PLIF/ TLIF cages. In their study, the maximum restoration of the disc height was 30\% which is lesser than what can be achieved through anterior approaches. Another potential advantage of larger cage is that it spans the vertebral endplate and rests on cortical rim whereas smaller posterior cage rests on cancellous bone surface and will have higher rates of cage sinkage which is particularly of concern in osteoporotic bones ${ }^{8)}$.

Various studies have shown efficacy of indirect neural decompression by analyzing clinical as well as radiological outcomes (Table 5).

Above table shows in our study that we could achieve better indirect decompression in terms of better radiological outcomes. All the patients were benefited with indirect decompression except in the series of Oliveira et al., two patients had requirement of direct decompression. This was observed because of loss of disc height secondary to cage subsidence due to stand alone cages. However, it is general recommendation to supplement the fixation to cage placement to avoid cage subsidence ${ }^{31)}$.

Another important question is the limitation of indirect decompression. In our study we counselled the patients preoperatively regarding the need of direct decompression in case of unresolved symptoms. Many times the patients do not show clinical symptoms as per the amount of stenosis seen radiologically or vice versa. So it is difficult to judge how much radiological indirect decompression is required for given patient. To find out if there is any correlation with amount of indirect decompression and clinical improvement, we had divided our patients into two groups, based on Schizas grading of lumbar canal stenosis. Group 1 with class A and B patients showed less improvement radiologically in spinal canal area postoperatively as compared to group 2 who had class C and D patients. However, clinically both the groups showed excellent to good outcomes. Authors tended to avoid this procedure in patients of Schizas grade D stenosis, except for 4 cases.

There were several limitations of our study. The sample size was smaller. Also, it being a retrospective study, there was selection bias regarding whom we chose for the procedure. We were inclined to avoiding OLIF in patients having severe lumbar canal stenosis (Schizas grade D) and listhesis grade 3 or more. Long term follow-up is mandatory for the evaluation of maintenance of reduction and clinical improvement apart from fusion status.

\section{CONCLUSION}

* OLIF is an effective means of indirect decompression in degenerative lumbar spine disease.

* Clinically and radiologically, there was statistically significant improvement postoperatively after indirect decompression.

* It cannot be proven from this study whether indirect decompression is effective for patients with Schizas grade D stenosis.

* None of the patients in this study required direct decompression after OLIF.

\section{REFERENCES}

1. Verbiest H: A radicular syndrome from developmental narrowing of the lumbar vertebral canal. J Bone Joint Surg Br 36:230237, 1954

2. Yong-Hing K, Kirkaldy-Willis WH: The pathophysiology of degenerative disease of the lumbar spine. Orthop Clin North Am 14:491-504, 1983

3. Fujiwara A, Tamai K, An HS, Lim TH, Yoshida H, Kurihashi A, Saotome K: Orientation and osteoarthritis of the lumbar facet joint. Clin Orthop Relat Res 385:88-94, 2001

4. Dino Samartzis, et al: Critical values of facet joint angulation and tropism in the development of lumbar degenerative spondylolisthesis: An international, large-scale multicenter study by the AOSpine Asia Pacific Research collaboration consortium. Global Spine J 6:414-421, 2016

5. Toyomi Yoshiiwa, Masashi Miyazaki, Naoki Notani, Toshinobu Ishihara, Masanori Kawano, Hiroshi Tsumura: Analysis of the relationship between ligamentum flavum thickening and lumbar segmental instability, disc degeneration, and facet joint osteoarthritis in lumbar spinal stenosis. Asian Spine J 10(6):11321140, 2016 
6. Toyomi Yoshiiwa, Masashi Miyazaki, Masanori Kawano, Shinichi Ikeda, Hiroshi Tsumura: Analysis of the relationship between hypertrophy of the ligamentum flavum and lumbar segmental motion with aging process. Asian Spine J 10(3):528-535, 2016

7. Inoue S, Watanabe T, Hirose A, Tanaka T, Matsui N, Saegusa $\mathrm{O}$, et al: Anterior discectomy and interbody fusion for lumbar disc herniation. A review of 350 cases. Clin Orthop Relat Res 183:22-31, 1984

8. Leonardo Oliveira, Luis Marchi, Etevaldo Coutinho, Luiz Pimenta: Radiographic assessment of the ability of the extreme lateral interbody fusion procedure to indirectly decompress the neural elements. SPINE Volume 35, Number 26S, ppS331-S337, 2010, Lippincott Williams \& Wilkins A.

9. Christopher K. Kepler, Amit K. Sharma, Russel C. Huang, Dennis S. Meredith, Federico P. Girardi, Frank P. Cammisa Jr, et al: Indirect foraminal decompression after lateral transpsoas interbody fusion. J Neurosurg Spine 16:329-333, 2012

10. Kim NH, Kim DJ: Anterior interbody fusion for spondylolisthesis. Orthopedics 14:1069-1076, 1991

11. Kim NH, Kim HK, Suh JS: A computed tomographic analysis of changes in the spinal canal after anterior lumbar interbody fusion. Clin Orthop Relat Res 286:180-191, 1993

12. Kim NH, Lee JW: Anterior interbody fusion versus posterolateral fusion with transpedicular fixation for isthmic spondylolisthesis in adults. A comparison of clinical results. Spine 24:812816, 1999

13. Kozak JA, Heilman AE, O’Brien JP: Anterior lumbar fusion options. Technique and graft materials. Clin Orthop Relat Res 300:45-51, 1994

14. Kuslich S, Gunzburg R, Szpalski M: The utility of interbody arthrodesis using BAK cages in treatment in lumbar spinal stenosis. Philadelphia, PA: LWW; 2000:287-301.

15. Lee SH, Choi WG, Lim SR, et al: Minimally invasive anterior lumbar interbody fusion followed by percutaneous pedicle screw fixation for isthmic spondylolisthesis. Spine J 4:644-649, 2004

16. Takahashi $\mathrm{K}$, Kitahara $\mathrm{H}$, Yamagata $\mathrm{M}$, et al: Long-term results of anterior interbody fusion for treatment of degenerative spondylolisthesis. Spine 15:1211-1215, 1990

17. Vamvanij V, Ferrara LA, Hai Y, et al: Quantitative changes in spinal canal dimensions using interbody distraction for spondylolisthesis. Spine 26:E13-18, 2001

18. D’Mitri A, Sofianos, Michael R. Brisen, Joshua Abrams, Alpesh A. Patel: Complications of the lateral transpsoas approach for lumbar interbody arthrodesis: A case series and literature review: Clin Orthop Relat Res 470:1621-1632, 2012 DOI 10.1007/ s11999-011-2088-3

19. Silvestre C, Mac-Thiong JM, Hilmi R, Roussouly P: Complications and morbidities of mini-open anterior retroperitoneal lumbar interbody fusion (Oblique Lumbar Interbody Fusion) in 179 patients. Asian Spine J 6:89-97, 2012

20. Mayer HM: A new microsurgical technique for minimally invasive anterior lumbar interbody fusion. Spine (Phila Pa 1976) 22:691-699, 1997

21. Dooris AP, Goel VK, Grosland NM, et al: Load-sharing between anterior and posterior elements in a lumbar motion segment implanted with an artificial disc. Spine 26:E122-129, 2001

22. White AA, Panjabi MM: Clinical biomechanics of the spine. 2ed. Philadelphia, PA: Lippincott Williams \& Wilkins; 1990: 92-111.

23. Bergey DL, Villavicencio AT, Goldstein T, et al: Endoscopic lateral transpsoas approach to the lumbar spine. Spine 29:16811688, 2004

24. Cummock MD, Vanni S, Levi AD, et al: An analysis of postoperative thigh symptoms after minimally invasive transpsoas lumbar interbody fusion. J Neurosurg Spine 15:11-18, 2011

25. Constantin Schizas, Nicolas Theumann, Alexandre Burn, Rosamond Tansey, Douglas Wardlaw, Francis W. Smith, et al: Qualitative grading of severity of lumbar spinal stenosis based on the morphology of the dural sac on magnetic resonance images. SPINE 35(21):1919-1924, 2010

26. Elowitz EH, Yanni DS, Chwajol M, Starke RM, Perin NI: Evaluation of indirect decompression of the lumbar spinal canal following minimally invasive lateral transpsoas interbody fusion: radiographic and outcome analysis. Minimally Invasive Neurosurg 54:201-206, 2011

27. Groth AT, Kuklo TR, Klemme WR, Polly DW, Schroeder TM: Comparison of sagittal contour and posterior disc height following interbody fusion: threaded cylindrical cages versus structural allograft versus vertical cages. J Spinal Disord Tech 18: 332-336, 2005

28. Jun Sato, et al: Radiographic evaluation of indirect decompre ssion of mini-open anterior retroperitoneal lumbar interbody fusion: oblique lateral interbody fusion for degenerated lumbar spondylolisthesis. Eur Spine J DOI 10.1007/s00586-015-4170-0

29. Shunsuke Fujibayashi, Richard A. Hynes, Bungo Otsuki, Hiroaki Kimura, Mitsuru Takemoto, Shuichi Matsuda: Effect of indirect neural decompression through oblique lateral interbody fusion for degenerative lumbar disease. SPINE 40(3):E175-E182, 2015 Wolters Kluwer Health.

30. Marjan Alimi, et al: Radiological and clinical outcomes following extreme lateral interbody fusion. J Neurosurg Spine 20:623-635, 2014

31. Marchi L, Abdala N, Oliveira L, et al: Radiographic and clinical evaluation of cage subsidence after stand-alone lateral interbody fusion. J Neurosurg Spine 19:110-118, 2013 